\title{
Compétence d'interaction et négociation du sens dans une activité de littératie entre pairs en immersion française en Colombie-Britannique (Canada)
}

\author{
CÉCILE SABATIER BULLOCK \\ Simon Fraser University
}

\begin{abstract}
Résumé
En contexte d'apprentissage du français langue seconde, la compétence d'interaction est une compétence clé à construire. Elle repose sur l'idée que l'apprenant communique et interagit avec ses interlocuteurs, pour la construction des savoirs langagiers et disciplinaires. Alors que les interactions entre enseignant et élèves font l'objet de nombreux travaux, le travail entre pairs reste encore opaque. À partir d'une activité de littératie entre élèves d'immersion sixième année, et en nous appuyant sur une approche sociolinguistique, il s'agit de montrer les rouages du travail collaboratif pour cerner les stratégies linguistiques et cognitives employées par les apprenants dans la résolution d'une tâche.
\end{abstract}

\section{Mots clés}

Littératie, compétence d'interaction, apprentissage d'une langue seconde, translanguaging, immersion française, Colombie-Britannique/Canada

\section{Introduction}

Lieu ordinaire et familier pour les enseignants et les élèves, la classe est un lieu complexe dès lors qu'il s'agit d'observer les processus d'acquisition et les processus de socialisation qui y prennent place. En classe, se construisent interactivement des rapports aux savoirs, des modes de relations entre chacun des acteurs de la relation didactique (enseignant et élèves, élèves entre eux) et des modes de compréhension du monde. Dans une perspective interactionniste de l'acquisition (voir Pekarek Doehler, 2000), l'aspect spécifique des apprentissages (qu'ils relèvent d'une langue ou d'une discipline) conduit à définir la classe comme un contexte d'appropriation et un espace de rencontres entre plusieurs interlocuteurs. Ces derniers sont engagés dans un processus commun d'apprentissage organisé autour d'objectifs à atteindre et d'activités pédagogiques partagées, et régi par des formats - au sens de Bruner (1985) - d'interactions que l'on peut dégager pour mettre en avant les dynamiques à l'œuvre dans la construction des savoirs. La classe devient intrinsèquement le lieu institué de la parole pour apprendre et les interactions des lieux d'acquisition (Coste, 2002) qui rendent indissociables le langagier du cognitif.

En contexte d'apprentissage $d u$ français langue seconde, la compétence d'interaction devient tout aussi majeure à construire que les compétences linguistiques. Tandis que dans une approche globale de l'utilisation du langage (qui ne tient pas compte des contextes), quatre savoir-faire sont traditionnellement évoqués (parler, lire, écrire, écouter). Une approche désormais centrée sur l'usage du langage en situation de communication, et en lien avec les perspectives actionnelle et écologique en didactique des 
langues (Kramsch, 2008 ; Larsen-Freeman \& Cameron, 2008), met en exergue cette cinquième compétence.

Cette compétence repose sur l'idée que l'apprenant communique et interagit avec ses interlocuteurs, y compris pour la construction, le transfert et la fixation des savoirs langagiers et disciplinaires (Cekaite, 2007 ; Wolff, 2003 ; Krafft \& Dausendschön-Gay, 1994 ; Bigot, 2005). L'apprenant doit donc non seulement communiquer (oralement ou par écrit) mais il doit désormais gérer la cohérence de son propre discours et l'accorder à celui de son interlocuteur. Fasel Lauzon, Pekarek Doehler et Pochon-Berger (2009, p. 121) définissent d'ailleurs la compétence d'interaction comme une compétence qui « intègre des ressources linguistiques, discursives, sociolinguistiques et interactionnelles, lesquelles sont indissociablement liées les unes aux autres ».

Afin d'aider l'apprenant de langue seconde (L2) à développer son langage, à l'utiliser de manière adéquate et à prendre sa place au sein d'une interaction qui se déroule dans un contexte de communication le plus souvent exolingue, une importance particulière doit donc désormais être apportée à la manière dont l'organisation des discours et des conversations se déroulent (Skogmyr Marian, Petitjean \& Pekarek Doehler, 2017). Arguant des enjeux cognitifs de la capacité à interagir dans les apprentissages (Hall \& Verplaetse, 2000 ; Halté, 1999), notre contribution a pour visée de souligner à quel point les échanges verbaux interviennent de façon centrale dans la construction des apprentissages. Mais alors que les interactions entre enseignants et élèves font l'objet de nombreux travaux (Claessens, van Tartwijk, van der Want, Pennings, Verloop, den Brok \& Wubbels, 2017 ; Haj-Broussard, Beal \& Boudreaux, 2017 ; Pennings, Brekelmansa, Sadlerb, Claessens, van der Want\& van Tartwijk, 2018 ; parmi d'autres), et que leurs mécanismes sont désormais bien connus (Cicurel, 2002 ; Bigot, 1996), il n'en va pas de même pour le travail en groupes, lequel «constitue un cadre psychosocial fort différent du travail en classe structuré et dirigé par l'enseignant » (Lusetti, 2004, p. 167).

Or, le travail en pairs est une des façons de travailler in situ la compétence d'interaction car il s'agit pour les élèves de négocier, de faire entendre leur point de vue, de réfuter des opinions, tout en réalisant une tâche. Dans ces négociations qui se donnent à voir entre chacun des membres du groupe, se révèlent les rôles et les identités que les interlocuteurs en présence endossent volontairement ou que leurs camarades leur assignent.

À partir d'un corpus recueilli dans une classe de sixième année d'un programme d'immersion précoce ${ }^{1}$ en français en Colombie-Britannique (province anglophone de l'ouest du Canada où le français est en situation minoritaire), nous nous proposons donc de donner à voir la manière dont se déroulent les interactions au sein de groupes d'élèves engagés dans la négociation du sens d'une histoire à raconter puis à écrire. Ce faisant, apparaîtra la façon dont chacun des élèves développent une compétence interactionnelle en L2 ainsi que la manière dont ils transfèrent les savoirs langagiers acquis (ou en cours d'acquisition) en langue seconde pendant la réalisation d'une activité de littératie.

\section{Cadrage théorique : Compétence d'interaction en L2, transferts interlinguistiques et collaboration entre pairs}

\footnotetext{
${ }^{1}$ Le programme d'immersion en français est un programme d'éducation bilingue dans lequel le français est utilisé pour enseigner différentes matières à des élèves dont il n'est pas la langue première. Dans le cadre d'une immersion précoce, l'enseignement débute dès la maternelle ou la première année de scolarisation.
}

\begin{tabular}{ll}
\hline Language and Literacy & Volume 21, Issue 3, 2019
\end{tabular}


La réflexion sur la compétence d'interaction en L2 est un champ qui a pris de l'importance dans les travaux sur l'acquisition des langues secondes, depuis l'apparition de la notion dans les écrits de Kramsch (1986, p. 370). Elle se situe dans une approche sociale de l'apprentissage qui considère que l'interaction structure les processus développementaux, lesquels sont fondamentalement situés dans le contexte social (Hall, 2004 ; Hellermann, 2008 ; Kasper, 2006 ; Mondada \& Pekarek Doehler 2001, 2004 ; Pekarek Doehler, 2000, 2002, 2005 ; Young, 2003).

A l'interface des travaux portant sur la compétence de communication telle que proposée par Hymes (1984), de ceux issus de l'analyse conversationnelle (Heritage, 1984), et de ceux en lien avec l'acquisition des langues secondes (Firth \& Wagner, 2007), la compétence d'interaction en L2 se définit comme la capacité qu'a un locuteur d'adapter ses productions langagières aux enjeux communicatifs et aux propriétés du contexte en mobilisant ses savoirs relatifs aux normes socioculturelles de son environnement de communication. Ce faisant, elle est une construction collective qui se matérialise à travers des procédures auxquelles ont recours les participants pour déployer une cognition socialement partagée de l'activité dans laquelle ils sont engagés (Markee, 2000 ; Mondada \& Pekarek Doehler, 2006 ; Pekarek Doehler, 2005 ; Schegloff, 2006).

Par le biais des interactions, et dans des modalités de classe qui favorisent la collaboration entre pairs, c'est l'ensemble des processus de transfert (psychologiques, mais aussi langagiers), que les élèves mettent en œuvre pour réaliser la tâche qui est la leur, qui est donné à voir. Pour Meirieu (1994), le transfert est ainsi la condition de l'apprentissage et le principe régulateur des pratiques pédagogiques. Une partie des travaux sur les transferts de connaissances en L2 portent attention à la manière dont en situation de contacts de langues, l'apprenant mobilise ses ressources langagières. Ainsi, au cours d'une activité de mise en mots lors d'une tâche de production orale spontanée, Trévisiol (2006) relève l'importance du rôle joué par les langues en présence et souligne la nécessité de s'intéresser aux choix des langues pour la réalisation de la tâche. Dans la lignée des travaux de Bialystok (1983) ou encore de Kellerman (1983), le recours aux autres langues des locuteurs constitue des stratégies de communication intentionnelles dans la mesure où les locuteurs y auraient recours pour palier des difficultés d'ordre lexical. Les travaux portant sur les transferts interlinguistiques dans le cadre de la recherche en acquisition des langues acceptent aujourd'hui l'idée que le recours par l'élève à l'ensemble des ressources langagières remplit une fonction centrale dans le processus d'acquisition d'une L2 (Perdue, 1995 ; Marquillo Larruy, 2003 ; Porquier \& Py, 2004 ; Véronique, 2007 ; Vogel, 1995, entre autres). Il ne s'agit plus alors d'évoquer des interférences entre systèmes langagiers mais plutôt d'observer comment, dans des interactions, les locuteurs de L2 mobilisent ces marques transcodiques (Marquillo Larruy, 2003) pour mener à bien des tâches communicatives.

L'intérêt pour les alternances de codes dans les discours des locuteurs apprenants d'une langue seconde ou tierce s'est traduit par l'émergence de la notion de « compétence plurilingue » dans les travaux francophones de didactique du français langue seconde (Coste, Moore \& Zarate, 2009 ; Castellotti \& Moore, 2015) et par celle du concept de «translanguaging » (voir entre autres García, 2009 ; García \& Li, 2013 ; García \& Kleyn, 2016 ; Creese \& Blackledge, 2010) dans les travaux portant sur les pratiques discursives des élèves bi-/plurilingues pour saisir leurs dynamiques d'apprentissage langagier. Ces deux notions conceptuelles renvoient ainsi aux conceptualisations du bilinguisme fondées 
sur les usages plutôt que sur l'idée de maitrise d'une langue (Grosjean, 1984 ; Lüdi \& Py, 2003), ainsi qu'à la notion post-structuraliste d'identités plurielles (Lahire, 1998), et mettent en avant les impacts éducatifs et pédagogiques quant à la prise de conscience, par l'individu, de la richesse de son répertoire linguistique (García \& Kano, 2014 ; García \& Sylvan, 2011 ; Litalien, Moore \& Sabatier, 2012).

Afin de mettre en lumière cette richesse, ainsi que les procédés stratégiques déployés par les apprenants, le travail entre pairs est par conséquent une façon de travailler la compétence d'interaction en situation scolaire. En lien avec la perspective socioculturelle et socioconstructiviste, Dagenais, Day et Toohey (2006), dans leur article sur les pratiques de littératie en contexte d'immersion française, affirment que ce serait grâce à la «participation à des activités communes et en utilisant des ressources spécifiques qu'un individu apprend » (p. 207 ; notre traduction). Selon elles, le travail collaboratif permet aux apprenants de puiser dans leur propre potentiel ainsi que dans celui de leurs coéquipiers pour construire des savoirs. Pour Rogoff (1990), la participation conjointe à une activité de résolution de problème favorise également la co-construction de nouvelles connaissances collectives, par le biais de l'étayage par les pairs. Dans cette optique, la collaboration et le travail avec les pairs présentent des avantages cognitifs comme le soulignent Halté (1999) ou Swain \& Lapkin (1998). Et pour Donato (2004), la collaboration participe même à transformer la place des individus à l'intérieur des groupes de pairs en en faisant des participants actifs qui contribuent à élargir les pratiques communicatives de ces dits groupes.

Lusetti (2004) témoigne d'ailleurs des différents types de coopération qui se mettent en place dans une activité de groupe. Dans ces négociations qui se donnent à voir entre chacun des membres du groupe, et que Swain (2001) qualifie de dialogue collaboratif dans la mesure où les apprenants sont engagés dans une résolution de problème au cours d'une tâche à accomplir, se révèlent également les rôles et les identités des interlocuteurs. L'attention portée aux rôles revendiqués par les apprenants en situation de classe permet de voir dans quelle mesure les dimensions sociales de l'interaction en classe ont été comprises et acquises par ces derniers.

\section{Ancrages méthodologiques}

Dans la perspective actionnelle de l'enseignement-apprentissage des langues aujourd'hui (Bento, 2013), associée à la notion de tâche issue du concept de Task-Based Learning tel que proposé par Ellis (2003) ou Nunan (2004), la communication langagière est un des moyens au service de l'action collective. Or la recherche en acquisition des L2 souligne le besoin de mieux connaître le développement interactif des apprenants. Par l'authenticité de la communication et des interactions qui prennent de facto place dans la salle de classe, puisque l'apprenant doit réaliser des tâches proposées par l'enseignant, la situation d'enseignement-apprentissage est donc orientée vers des situations de communication les plus authentiques possibles.

L'approche de recherche qui est la nôtre ici s'inscrit dans cette optique. Elle prend appui sur une approche littératiée pour l'enseignement du français langue seconde (Anderson, Carr, Lewis, Salvatori \& Turnbull, 2008). Dans une perspective microsociologique, les élèves d'une classe de sixième année d'immersion précoce en français de la grande banlieue de Vancouver (Colombie-Britannique), regroupés en groupes de pairs par l'enseignante suite à un tirage au sort (pratique courante des salles de 
classe britanno-colombiennes pour former des groupes), vont négocier le sens d'une histoire à partir d'une collection d'albums sans mots ${ }^{2}$. Ces derniers permettent de travailler le récit oral et la macrostructure de la narration à partir d'une trame prototypique du récit narratif (un cadre, un élément déclencheur, des tentatives de résolution et la résolution du problème), ainsi que les dimensions plus linguistiques telles que les connecteurs, la temporalité, etc. La collection est couramment utilisée dans les travaux portant sur le développement du langage des enfants de par le monde (Heilmann, Rojas, Iglesias \& Miller, 2015; Hilaire-Debove \& Kern, 2013). Enfin, l'activité ici analysée fait partie d'une recherche de plus grande ampleur qui a porté sur le développement de la compétence en langue seconde chez des élèves scolarisés en immersion en français de la deuxième à la douzième année. Dans le contexte de cette classe, le français est certes la L2 à apprendre dans le contexte scolaire, mais au regard des répertoires langagiers déclarés des élèves, c'est aussi la quatrième, voire cinquième ou sixième langue acquise après les langues familiales (Urdu, Punjabi, entre autres) et la langue de l'environnement (Anglais).

L'analyse des données s'inscrit, quant à elle, dans l'analyse conversationnelle qui adopte une démarche empirique et procède à l'analyse de données authentiques dans une perspective émique. L'analyse des données permet ainsi de dégager des phénomènes dont la description permet la mise en lumière des stratégies utilisées par les participants pour accomplir conjointement une action au fur et à mesure que se déroulent les échanges. Ces derniers sont représentatifs et caractéristiques de la machinerie des tours de parole ainsi que des principes qui organisent l'interaction et qui régissent la réalisation de la tâche ; ils font montre également des activités (socio)-cognitives qui sont mobilisées par l'ensemble des apprenants pour mener à bien la tâche. Pour ce faire, après avoir obtenu les consentements éthiques pour mener à bien la recherche, et après avoir enregistré au moyen de dictaphones (un dictaphone par groupe d'élèves), les tours de paroles dans les différents groupes de pairs (numérotés de 1 à 6 et identifiés de G1 à G6) ont été transcrits. Ces tours de parole sont porteurs d'un numéro de ligne, ce qui facilite leur repérage dans le corpus. Au regard des conventions de transcription, les marques transcodiques sont annoncées par l'emploi de l'italique ; aucune ponctuation n'a été utilisée ; le point est ici simplement un marqueur de pause. Enfin, les élèves sont identifiés par des numéros afin de respecter l'anonymat des participants.

Les questions qui ont guidé notre analyse sont les suivantes :

- Comment se déploie en situation de collaboration la compétence d'interaction en français langue seconde dans une classe en immersion?

- Quelles pratiques et stratégies communicatives et interactives les élèves mettent-ils en place plus précisément lors d'une activité de littératie qui demande la négociation du sens d'une histoire?

- Quels rôles et identités les élèves révèlent-ils au fur et à mesure des échanges et au regard de la construction des savoirs?

- Quelles mobilisations et utilisations des ressources langagières les élèves dans les groupes de pairs donnent-ils à voir, notamment au regard des langues qui composent leurs répertoires verbaux ?

\footnotetext{
2 Il s'agit de la collection des albums de Mercer Mayer : A boy, a dog and a frog (1967); Frog Where are you ? (1969); Frog on his own (1973); One frog too many (1975). Ces albums sont publiés par Dial Books, une division de Penguin Young Readers Group, New York.
} 
Elles ont permis de dégager successivement a) les différentes manières dont chacun des groupes a pris en charge la gestion de la tâche à accomplir (à savoir, raconter une histoire) en termes d'organisation des interactions entre élèves, b) les activités (socio)-cognitives qui ont été mises en œuvre par les élèves au cours de la négociation du sens de l'histoire à raconter, ainsi que c) les limites de la situation didactique au regard de la mise en œuvre de la compétence d'interaction. Les questions ont également conduit à s'interroger sur le rôle et la place des langues en contact dans les répertoires verbaux des élèves au regard des apprentissages scolaires.

\section{Résultats}

Dans le cadre de l'étude des interactions sociales et de la compétence d'interaction dans sa dimension langagière (laquelle renvoie à la compétence en langue), le matériel verbal à disposition permet d'observer le déploiement de la compétence dans l'interaction. La compétence observée est ainsi toujours une "compétence en action" (Pekarek Doehler, 2006), située et accomplie collectivement, à travers les ajustements mutuels des participants.

En réponse à la question portant sur la manière dont les élèves communiquent entre eux au cours de cette activité de négociation du sens d'une histoire à raconter, il apparaît que dans l'ensemble des groupes, deux types d'épisodes interactionnels (Guerrerro \& Villamil, 1994) prennent place. Le premier d'entre eux renvoie aux séquences d'interactions qui sont centrées sur la tâche à accomplir, comme l'illustre l'exemple ciaprès :

41. E3: okay un garçon va au pêche avec ses animaux pour marcher 42. E1: ça c'est une promenade promenade (Groupe 4)

Le second épisode interactionnel est celui à propos de la tâche avec notamment un rappel de la consigne de parler en français :

83. E1 : parlez français (Groupe 4)

74. E1: il faut parler le français . quand le garçon était dans la forêt (Groupe 2)

Il convient de noter que ce que certains auteurs ont nommé « la parole buissonnière » (Daunay et al., 1995) pour caractériser des interactions hors tâche, et qui le plus souvent manifeste la difficulté pour des élèves à poursuivre une tâche, n'est pas présente dans le corpus, sauf lorsque les élèves ont accompli le travail demandé. L'habitude de travail en groupes par les élèves, l'implication des élèves dans la tâche et l'enregistrement des interactions peuvent expliquer cette absence. Quant à ce qui est de la difficulté de la tâche à proprement dite, nous verrons dans la quatrième partie, comment les groupes sont parvenus à contourner cet écueil.

La gestion de la tâche ou les principes organisationnels des interactions dans les groupes de travail

En portant l'attention sur les schémas (ou «patterns ») interactionnels (Garfinkel, 1967), tels que décrits par l'analyse conversationnelle, la gestion de la tâche par l'ensemble 
des groupes suit une organisation similaire : ouverture de la conversation (salutations et histoire à raconter ; 2. E1: hello, 3. E2: bonjour, 4. E3: alors one frog too many (Groupe 1)); construction du sens de l'histoire à partir de son déroulement selon le principe d'une image un thème (21. E1: on a pas fait page 4 yet (Groupe 5)) puis clôture de la conversation (242. E2: maintenant je vais write up (Groupe 1)). Chacune des interactions dans les différents groupes débute ainsi par le rappel de l'objectif de la tâche : raconter une histoire. Dans certains groupes, cette ouverture est initiée par l'enseignante puis rappelée par un élève qui va adopter soit un rôle de médiateur soit un rôle de leader au sein du groupe.

Lors de la phase de la négociation du sens de l'histoire, ensuite, dans le groupe 6 par exemple, chacun des élèves parle à tour de rôle sur une page de l'histoire à raconter, donnant ainsi à voir un principe d'organisation des interactions selon une structure linéaire en lien avec la narration de l'histoire appuyée sur les images du support. Ces principes organisationnels des interactions dans les groupes de pairs reposent sur les savoirs et savoir-faire en L2 des élèves et mettent en lumière un cadre participatif à l'intérieur duquel les élèves agissent ensemble et communiquent sur la tâche et autour de la tâche, un cadre à l'intérieur duquel, autrement dit, les élèves se mettent en place et font le choix de parler ou de se taire, de proposer des idées ou de confirmer ce qui est avancé.

Ce cadre participatif (y compris dans les interactions conflictuelles) prend appui sur une histoire interactionnelle entre les membres du groupe, laquelle se donne à son tour à voir à travers les marqueurs du discours qui ponctuent et témoignent de l'approbation (ou non) du groupe (okay; oui ; okay we go okay; yeah ; rires de connivences ; non), dans les tours de parole respectés et autorégulés ou encore dans la prise de places et les rôles auto et hétéro-assignés. Ainsi, dans quatre des six groupes, un élève adopte le rôle de leader qui prend en charge de rappeler la consigne et de rappeler l'usage du français (34. E2: il faut parler français (Groupe 4)). Dans le groupe 2, c'est un élève qui raconte l'histoire que les autres membres du groupe acquiescent, et, qui s'assure de sa cohérence (12. E2: We got this already 13. E3: on what 14. E3: on here you see (Groupe 2)). Cet élève apparait comme un « leader cognitif » (Accardi, Moussu \& Raby, 2008), car il est l'élève le plus compétent et le plus à l'aise pour interagir en L2 et mener à bien la tâche à accomplir dans la langue seconde.

\section{La réalisation de la tâche et les activités (socio)-cognitives}

Les activités (socio)-cognitives qui se déploient dans la réalisation de la tâche pour envisager la construction et le développement de la compétence d'interaction en L2 sont d'abord régulées par les représentations que les élèves se font de la tâche :

\section{E3: we should all work (..) it's really hard work (Groupe 2)}

Pour certains des groupes, le travail est assez vite terminé. On dit ce qu'on voit. Une lecture de l'image s'opère ainsi, sans véritable souci d'un récit narratif enrichi. Dans ces groupes, chacun des pairs a une image à raconter selon l'équation : un pair $=$ un tour de parole = une image. Dans le groupe qui a le plus difficulté à rester sur la tâche, la distribution et l'organisation des tours de parole pour construire ensemble le sens de l'histoire est plus compliquée. Le discours est, ici, de nombreuses fois, parsemé de «shut up » lorsqu'un élève parle quand ce n'est pas son tour. Un groupe cependant, s'attache, lui, à imaginer une histoire complète et la négociation de la signification de la tâche permet 
alors pour ce groupe l'élaboration de buts communs pour l'histoire à raconter. Ce groupe saisit l'opportunité de raconter une histoire par delà l'image et mobilise son imagination s'engageant alors dans des moments de négociation plus fréquents :

178. E2: why not talk of that 179. E3: we have to maybe we have to do that (rires) okay that's such a good point (Groupe 2)

Dans les interactions entre les élèves au sein des différents groupes, il apparaît ainsi que négocier le sens de l'histoire consiste le plus souvent à amener son interlocuteur à apporter une information ou à la modifier. Négocier le sens du message avec son interlocuteur, c'est autrement dit être capable de répéter une information pour s'assurer qu'elle est la bonne et qu'elle est partagée par le groupe. Cela consiste également à vérifier que les éléments de l'histoire ne sont pas répétés afin de garantir sa cohérence. Ces interactions sont des formes de négociation puisqu'il s'agit pour les élèves engagés dans la tâche et la communication de s'assurer que le récit narratif progresse de manière adéquate. À d'autres occasions, négocier le sens de l'histoire revient à demander un mot dans la langue seconde ou à revenir sur une information qui n'a pas été comprise :

24. E2: non c'est quoi tortoise l'autre jour il y a le tortoise, 25. E3:

c'était tortue, 26. E2: c'est vrai, 27. E3: yeah c'est vrai (Groupe 4)

Dans cette interaction, les élèves sont des interlocuteurs actifs, car ils sont capables de solliciter l'aide linguistique de leurs pairs une fois leurs propres ressources langagières épuisées, puis de valider le mot proposé comme étant bien le mot attendu. Au regard de la question de la négociation du sens, il semble que la tâche permet d'engager les élèves en leur fournissant l'opportunité d'agir comme des négociateurs quant au vocabulaire à mobiliser pour raconter l'histoire. Ici, se donne donc à voir la convocation des acquis lexicaux par le biais de l'étayage du vocabulaire par les pairs. L'exemple suivant est encore plus illustratif de cet étayage linguistique par et entre les pairs :

40. E11. alright le grenouille c'est un frog 41. E3: okay un garçon va au pêche avec ses animaux pour marcher, 42. E1: ça c'est une promenade une promenade, 43. E3: avec deux garçons, 44. E2: il va à la pêche pour faire un promenade et les et il fait, 45. E3: une promenade, 46. E2: Memory you are a walking dictionary (Groupe 5)

Dans l'exemple ci-dessus, apparaît en effet la manière dont les élèves qui connaissent le vocabulaire approprié en français adoptent le rôle de «passeurs de langue » pour leurs camarades. Dans les tours de parole du groupe 5, se dessine clairement la construction d'un contexte d'apprentissage potentiellement acquisitionnel (De Pietro, Matthey \& Py, 1989) dans la mesure où les interactions mettent à jour un format interactif susceptible de faciliter l'acquisition lexicale du mot «promenade ». Dans l'ensemble du corpus, ces séquences potentiellement acquisitionnelles sont surtout situées au niveau du lexique, comme cet autre exemple le montre à partir de la discussion entre deux élèves: 
72. E3: [il] se lave il voit le grenouille 73. E1: non il se lève 74. E3:

il se lave 75. E1: il se lève à la surface il se lève 76. E3: il se lève sur la surface 77. E1: oui (Groupe 3)

Dans cet échange, les deux élèves négocient lequel du mot «se lève » ou «se lave », mots phonétiquement proches, est le mot correct pour raconter l'histoire. Ils négocient la cohérence du vocabulaire en lien avec ce qui est montré sur l'image. Une sorte de joute verbale implicite s'engage entre les deux élèves après que E1 a repris E3 quant au mot à employer. Sans entrer pour autant dans une séquence explicative qui a pour finalité de faire comprendre quelque chose à quelqu'un (Fasel Lauzon, 2009), E1 conduit E3 à intégrer une nouvelle connaissance lexicale, témoignant du potentiel d'apprentissage de cet échange. Le réemploi du mot par E3 et sa validation par E1 mettent un terme à cet épisode interactionnel autour du lexique.

\section{Limites des résultats}

La situation didactique constituée par la tâche de littératie à réaliser en groupe de pairs a ritualisé la prise de parole des élèves. Cette dernière est marquée par les consignes de l'activité. On y retrouve ainsi les traces des interactions de classe régulières entre enseignant et élèves puisque dans chacun des groupes, un des élèves adopte à un moment ou l'autre le rôle d'enseignant. Cela conduit à une séquence didactique qui est hautement sensible au contexte d'apprentissage et au niveau de langue à disposition. Le dialogue qui s'instaure dans les groupes est par conséquent un dialogue fortement ritualisé.

Toufefois, dans l'ensemble des groupes, on assiste également à une déritualisation $\mathrm{du}$ format interactif, notamment par le recours à une autre des langues qui compose le répertoire verbal des apprenants. Selon Moore et Simon (2002), les apprenants redessinent ici, à leur façon, et selon leurs propres finalités de communication, les enjeux de l'interaction didactique, en particulier, et de l'interaction sociale plus largement. En mobilisant l'ensemble des ressources de leur répertoire communicatif, ils font montre des stratégies de communication dont ils disposent.

\section{Rôle de la L1 dans l'apprentissage de la L2 : stratégie de compensation ou émergence de la compétence d'interaction plurilingue?}

Ces stratégies de communication mettent en lumière comment les difficultés cognitives et linguistiques de la tâche à accomplir sont surmontées par les élèves. Ainsi, la production du récit à raconter en mode bilingue, avec la présence de l'anglais dans tous les groupes, apparaît rapidement comme une façon de maintenir l'engagement cognitif dans la tâche tout en contournant les défis linguistiques que représente raconter une histoire en langue seconde.

Ce transfert interlinguistique, indubitablement lié au niveau d'acquisition de la langue 2, apparaît dans un premier temps comme autant de stratégies de compensation, et ce notamment dans les groupes les plus faibles linguistiquement. Le recours à l'anglais dans ces groupes permet en effet d'élucider le sens de l'histoire à négocier pour mieux ensuite accomplir la tâche à l'oral en contournant l'obstacle de la gestion conjointe du linguistique et du discursif.

Toutefois, en s'inscrivant dans les travaux sur les marques transcodiques produites dans les parlers bilingues (Lüdi, 1995 ; Py, 1991 ; Lüdi \& Py, 2003), ce recours à l'autre 
langue du répertoire langagier des apprenants apparaît également dans les groupes les plus forts linguistiquement, non plus comme des interférences, mais comme le recours à une compétence d'interaction plurilingue en lien avec le type de tâche à réaliser et les modalités communicationnelles utilisées par les élèves. Dans le cadre de la négociation du sens, Kramsch (1986), qui a été une des premières à souligner l'importance en L2 de se démarquer d'un modèle ou d'une conception déficitaire des productions des apprenants de L2, insiste d'ailleurs qu'en étudiant le développement de la compétence interactionnelle chez les apprenants de L2, cela permet de se concentrer davantage sur les habiletés de ces derniers à établir une compréhension mutuelle des intentions de communication.

Dans l'extrait ci-après, le recours aux deux langues en alternance souligne l'effort des élèves à mettre en mots dans la langue-cible leur histoire. Ils mobilisent la langue anglaise à la fois comme procédés d'appui, d'aide, de pointage d'un manque linguistique, tout en marquant la volonté de maintenir l'échange malgré tout. Ainsi, l'extrait illustre précisément comment le recours à l'alternance codique mais aussi le recours à l'ensemble des ressources langagières des répertoires verbaux individuels permet, par-delà établir une compréhension commune de l'histoire à raconter, de mettre en lumière l'émergence de la compétence plurilingue chez les apprenants:

6. E3: no grenouille he wants to get a grenouille okay après il a tombé dans l'eau. Regarde (rires) il pleut, 7. E2. it looks like just a dog oh no grenouille, (..) 68. E2: what is he doing il voit le grenouille . Soudainement oh c'est mon he's already dans l'eau (Groupe 3)

Par compétence plurilingue, il faut entendre ce que Coste, Moore et Zarate (2009) ont défini comme la capacité pour un individu à mobiliser l'ensemble de ses ressources langagières pour communiquer: "Disposer de compétences dans plus d'un seul code linguistique, c'est pouvoir passer d'une langue à l'autre, selon les situations. Mais il arrive aussi que les plurilingues, entre eux, passent d'une langue à l'autre, parfois au sein d'un même énoncé. (..) Les plurilingues recourent au changement de langue - ou parler bilingue - de manière stratégique pour négocier du sens, porter des messages de contenu, donner des informations sur le locuteur, son identité sociale et culturelle, la place qu'il occupe dans la conversation, ou la nature de l'échange. » (2009, p. 18)

En caractérisant le discours des apprenants du groupe 3 de parler bilingue, cela implique de reconnaître leur maîtrise des systèmes langagiers en contact car chacun des idiomes en présence répond à ses propres règles et pouvoir passer d'une langue à l'autre comme ils le font «s'inscrit dans un fonctionnement social, qui régule ses modes d'apparition et les valeurs stratégiques qui s'y attachent. Le recours au parler bilingue dans les conversations entre pairs permet de focaliser doublement le message, par la mise en incise du contenu » (Coste, Moore \& Zarate, 2009, p. 19).

Le recours à l'alternance de codes, ainsi que les «pratiques discursives multiples dans lesquelles les bilingues s'engagent afin de donner un sens à leur monde bilingue» (Garcia, 2009, p. 45) lors de la production du récit à l'oral en mode bilingue, place dès lors les interactions entre ces élèves du côté du développement d'une compétence plurilingue, qui dans la perspective d'une tâche cognitive de négociation du sens, témoigne de la création d'une cohérence sémantique et grammaticale entre des éléments appartenant aux deux systèmes linguistiques différents. Ce faisant, les élèves d'immersion dans le cadre de 
cette activité de littératie font montre de l'émergence d'une compétence d'interaction plurilingue qui mobilise de manière dynamique l'ensemble de leurs ressources cognitives, linguistiques et discursives. Ils révèlent comment ils «font appel à différentes caractéristiques sociales dans un réseau homogène et complexe de multiples signes sémiotiques, à mesure qu'ils adaptent leur langage à la tâche qui les attend » (García \& Kano, 2014, p. 260-261, notre traduction). En mobilisant l'ensemble de leur répertoire linguistique dans la réalisation de la tâche, les élèves font la démonstration de ce qu'ils savent et peuvent faire au cours de leur apprentissage du français langue seconde.

\section{Conclusion}

Au final, l'étude des interactions au sein des groupes de pairs dans une activité de littératie permet de saisir les principes organisationnels des interactions dans le travail de groupes, ainsi que les activités (socio)cognitives qui se déploient dans la réalisation de la tâche pour envisager la construction et le développement de la compétence d'interaction en français langue seconde. L'analyse des modalités de travail en groupe pour la négociation du sens d'une histoire à raconter montre que les apprenants redéfinissent la tâche qui leur est proposée afin de se l'approprier ainsi que les moyens langagiers pour la mener à bien.

À l'intérieur de chacun de leur groupe, les élèves mettent en place un cadre interactionnel au sein duquel ils vont négocier le récit narratif en usant des ressources langagières qu'ils ont à disposition. Ce faisant, le choix des langues les conduit à alterner entre le français et l'anglais. Dès lors, comme le soulignait déjà Swain (2000, p. 60), le travail collaboratif autour de la tâche offre des opportunités pour l'apprentissage de la L2 dans la mesure où les élèves prennent conscience de leurs lacunes linguistiques lorsqu'ils essaient de construire du sens dans la langue seconde. Le travail de groupes leur permet également d'externaliser leur savoir langagier, de l'appliquer, de le questionner, voire de le réviser si nécessaire. Confrontés à des séquences potentiellement acquisitionnelles, les apprenants accroissent leurs connaissances dans et de la langue seconde selon leurs besoins.

Lorsque la gestion du linguistique et du discursif dans la tâche devient un obstacle infranchissable, celle-là est résolue par le recours au transfert interlinguistique puisque tous les groupes mènent la tâche à l'oral en alternant anglais et français. Ce transfert interlinguistique apparaît alors comme l'émergence de la compétence plurilingue chez des apprenants qui mobilisent plusieurs langues dans leurs répertoires verbaux pour composer un réseau sémiotique adapté à la situation de communication qui est la leur. L'analyse des formats d'interactions permet ainsi de souligner que, dans le cadre de cette conversation menée en mode bilingue, chacun des apprenants connaît les modalités de fonctionnement de l'interaction sociale et didactique.

L'étude des interactions révèle enfin les rôles et les identités que chacun des membres du groupe de pairs adopte au fil des interactions. De l'élève qui endosse le rôle de l'enseignant, à celui qui devient " passeur de langues » ou encore au « leader cognitif », chacun des apprenants au fil des interactions est conduit à prendre part à l'interaction selon un cadre social interactif ritualisé et implicite.

Au regard de l'enseignement-apprentissage du français dans un contexte de langue seconde (en immersion) mais également minoritaire (dans une province anglophone empreinte d'une grande diversité linguistique et culturelle), la mobilisation de l'ensemble 
des ressources langagières à disposition par les apprenants, mise en lumière par l'étude de la compétence d'interaction, conduit à (re)mettre en question la séparation des langues dans les contextes scolaires et à envisager la notion de translanguaging comme un concept central de l'enseignement-apprentissage de ces dernières, qu'elles soient langues premières, secondes ou tierces. Comme le mentionnent Turnbull (2016), García (2017) ou encore Gajo \& Steffen (2015) et Candelier \& Castellotti (2013), il s'agit, autrement dit, désormais, d'inscrire les démarches d'enseignement et d'apprentissage des langues dans le paradigme du bi/plurilinguisme. Celui-ci favorise le développement d'une compétence plurilingue qui prend appui sur les acquis linguistiques déjà-là des élèves et conduit à délaisser les représentations unicistes de la langue, du milieu d'enseignementapprentissage et des pratiques pédagogiques qui y prennent place, pour traiter le français en lien et en relation avec les autres langues en contact dans les répertoires verbaux des élèves (Sabatier Bullock, sous presse).

\section{Bibliographie}

Accardi, J., Moussu, M.-J. \& Raby, F. (2008). La dynamique motivante du groupe en L2 à l'école primaire: une étude de cas. Les langues modernes, 3, 44-52.

Anderson, B., Carr, W., Lewis, C., Salvatori, M. \& Turnbull, M. (2008). Effective literacy practices in FSL: Making connections. Toronto, ON: Pearson Canada.

Bento, M. (2013). Regards théoriques sur la perspective actionnelle dans l'enseignement des langues en France. Éducation et didactique, 7(1), 87-100.

Bigot, V. (2005). Négociation de la relation et processus d'appropriation en classe de langue. Acquisition et interaction en langue étrangère, (22), 17-43. Repéré à https://aile.revues.org/1716

Bigot, V. (1996). Converser en classe de langue: mythe ou réalité ? Dans F. Cicurel et E. Blondel (Eds.). La construction interactive du discours de la classe de langue (pp. 33-46). Paris, France: Presses Universitaires de la Sorbonne Nouvelle.

Bialystok, E. (1983). Some factors in the selection and implementation of communication strategies. Dans C. Faerch, and G. Kasper (Eds). Strategies in interlanguage communication (pp. 100-118). London, UK: Longman.

Bruner, J. (1985). The role of interaction formats in language acquisition. Dans J. P. Forgas (Ed.), Language and social situations (pp. 31-46). New York, NY: Springer.

Candelier, M. \& Castellotti, V. (2013). Didactique(s) du/des plurilinguisme(s). Dans J. Simonin \& S. Wharton, (Eds.), Sociolinguistique des langues en contact, modèles, théories. Dictionnaire encyclopédique des termes et concepts (pp. 293-318). Lyon, France: ENS-LSH Editions.

Castellotti, V. \& Moore, D. (2015). La compétence plurilingue et pluriculturelle: Genèse et évolutions d'une notion-concept. Dans P. Blanchet \& P. Chardenet (Eds.), Guide pour la recherche en didactique des langues et des cultures. Approches contextualisées (pp. 291-301). Paris, France: Éditions des archives contemporaines.

Cekaite, A. (2007). A child's development of interactional competence in a Swedish L2 classroom. The Modern Language Journal, 91(1). 45-62. Repéré à https://www.diva-portal.org/smash/get/diva2:248737/FULLTEXT01.pdf 
Cicurel, F. (2002). La classe de langue un lieu ordinaire, une interaction complexe. Acquisition et interaction en langue étrangère, (16), 145-164. Repéré à https://aile.revues.org/801

Claessens, L. C. A., van Tartwijk, J., van der Want, A. C., Pennings, H. J. M., Verloop, N., den Brok, P. J. \& Wubbels, T. (2017). Positive teacher-student relationships go beyond the classroom, problematic ones stay inside. The Journal of Educational Research, 110(5), 478-493.

Coste, D., Moore, D. \& Zarate, G. (2009). Compétence plurilingue et pluriculturelle. Strasbourg, France: Conseil de l'Europe. (Première édition : 1997)

Coste, D. (2002). Quelle(s) acquisition(s) dans quelle(s) classe(s)? Acquisition et interaction en langue étrangère, (16), 3-22. Repéré à https://aile.revues.org/747

Creese, A. \& Blackledge, A. (2010). Translanguaging in the bilingual classroom: A pedagogy for learning and teaching? The Modern Language Journal, 94(1), 103115.

Dagenais, D., Day, E. \& Toohey, K. (2006). A multilingual child's literacy practices and contrasting identities in the figured worlds of French immersion. International Journal of Bilingual Education and Bilingualism 9, 205-218.

Daunay, B., Delcambre, I., Marguet, S. \& Sauvage, C. (1995). Pratiques d'oral dans le travail de groupe. Recherches, (22), 169-203.

De Pietro, J.-F., Matthey, M. \& Py, B. (1989). Acquisition et contrat didactique : Les séquences potentiellement acquisitionnelles dans la conversation exolingue. Dans K. W. Phinainitisatra, T. Boontharm, P. Thipkong, S. Dejamonchai, F. Carral \& J.

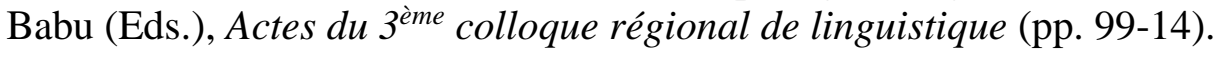
Strasbourg, France: Université des Sciences Humaines.

Donato, R. (2004). Aspects of collaboration in pedagogical discourse. Annual Review of Applied Linguistics, 24, 284-302.

Ellis, R. (2003). Task-based language learning and teaching. Oxford, UK: Oxford University Press.

Fasel Lauzon, V., Pekarek Doehler, S. \& Pochon-Berger, E. (2009). Identification et observabilité de la compétence d'interaction: Le désaccord comme microcosme interactionnel. Bulletin Suisse de Linguistique Appliquée, 86, 121-142.

Fasel Lauzon, V. (2008): Interactions et apprentissages dans des séquences d'explication de vocabulaire. TRANEL, 48, 83-104.

Firth, A. \& Wagner, J. (2007). Second/Foreign language learning as a social accomplishment: Elaborations on a reconceptualized SLA. The Modern Language Journal, 91, 800- 819.

Gajo, L. \& Steffen, G. (2015). Didactique du plurilinguisme et alternance de codes: le cas de l'enseignement bilingue précoce. La revue Canadienne des langues vivantes, 71(4), 471-499.

García, O. \& Kleyn, T. (Eds.) (2016). Translanguaging with multilingual students: Learning from Classroom Moments. New York, NY: Routledge.

García, O. \& Wei, L. (2014). Translanguaging: language, bilingualism and education. Basingstoke, UK: Palgrave Macmillan UK.

García, O. \& Kano, N. (2014). Translanguaging as process and pedagogy: Developing 
the English writing of Japanese students in the U.S. Dans J. Conteh \& G. Meier (Eds.), The multilingual turn in languages education: Benefits for individuals and societies (pp. 258-277). Clevedon, England: Multilingual Matters.

García, O. \& Sylvan, C. E. (2011). Pedagogies and practices in multilingual classrooms: Singularities in pluralities. The Modern Language Journal, 95(3), 385-400.

García, O. (2017). Reflections on Turnbull's reframing of foreign language education: Bilingual epistemologies. International Journal of Bilingual Education and Bilingualism, 20(1), 1-11.

García, O. (2009). Bilingual education in the 21st century: A global perspective. Malden, MA: Blackwell.

Garfinkel, H. (1967). Studies in ethnomethodology. Englewood Cliffs, NJ: Prentice-Hall. Grosjean, F. (1984). Le bilinguisme: vivre avec deux langues. Revue tranel, 7, 15-41.

Guerrerro, M. \& Villamil, O. (1994). Socio-cognitive dimensions of interaction in L2 peer revision. The Modern Language Journal, 78(4), 484-496.

Haj-Broussard, M., Beal, H.K.O. \& Boudreaux, N. (2017). Relating French immersion teacher practices to better student oral production. The Canadian Modern Language Review, 73(3), 319-342.

Hall, J., Hellermann, J. \& Pekarek Doehler, S. (Eds.) (2011). L2 Interactional competence and development. Clevedon, England: Multilingual Matters.

Hall, J. K. \& Verplaetse, L. (Eds.) (2000), Second and foreign language learning through classroom interaction. Mahwah, NJ: Lawrence Erlbaum.

Hall, J. K. (2004). Language learning as an interactional event. The Modern Language Journal, 88, 607-611.

Halté, J.-F. (1999). Les enjeux cognitifs des interactions. Pratiques, 103(1), 71-88.

Heilmann, J., Rojas, R., Iglesias, A. \& Miller, J. (2015). Clinical impact of wordless picture storybooks on bilingual narrative language production: A comparison of the 'Frog' stories. International Journal of Language \& Communication Disorders, 51(3), 1-7.

Hellerman, J. (2008). Social actions for classroom language learning. Clevedon, England: Multilingual Matters.

Heritage, J. (1984). Garfinkel and ethnomethodology. Cambridge, UK: Polity press.

Hilaire-Debove, G. \& Kern, S. (2013). Evaluation \& développement de la macrostructure du récit oral chez les enfants avec ou sans troubles du langage. A.N.A.E., 124, 110.

Kasper, G. (2006). Beyond repair: Conversation analysis as an approach to SLA. AILA Review, 19, 83-99.

Kellerman, E. (1983). Now you see it, now you don't. Dans S. Gass \& L. Selinker (Eds.), Language transfer in language learning (pp. 112-134). Boston, MA: Newbury House.

Klein, W. \& Perdue, C. (1992). Utterance structure: Developing grammars again. Amsterdam: John Benjamins.

Krafft, U. \& Dausendschön-Gay, U. (1994). Analyse conversationnelle et recherche sur l'acquisition. Bulletin VALS/ASLA, 59, 127-158.

Kramsch, C. (1986). From language proficiency to interactional competence. The Modern Language Journal, 70(4), 366-372.

Kramsch, C. (2008). Ecological perspectives on foreign language education. Language 
Teaching, 41(3), 389-408.

Lahire, B. (1998). L'homme pluriel: Les ressorts de l'action. Paris, France: Armand Colin.

Larsen-Freeman, D., \& Cameron, L. (2008). Complex systems and applied linguistics. Oxford, UK: Oxford University Press.

Litalien, R. J., Moore, D. \& Sabatier, C. (2012). Ethnographie de la classe, pratiques plurielles et réflexivité: pour une écologie de la diversité en contexte francophone en Colombie-Britannique. Revue canadienne de l'éducation, 35(2), 192-211.

Lüdi, G. \& Py, B. (2003). Être bilingue, 3e édition. Berne: Peter Lang.

Lüdi, G. (1995). Parler bilingue et traitements cognitifs. Intellectica, 1(20), 139-156.

Lusetti, M. (2004). Interactions verbales et gestion d'une tâche scolaire entre pairs. Dans A. Rabatel (Ed.), Interactions orales en contexte didactique (pp. 167-202). Lyon, France: Presses Universitaires de Lyon.

Markee, N. (2000). Conversation analysis. Mahwah, NJ : Erlbaum.

Marquillo Larruy, M. (2003). L'interprétation de l'erreur. Paris, France : Clé International.

Meirieu, P. (Ed.) (1994). Le Transfert de connaissances en formation initiale et en formation continue. Actes du colloque organisé à l'université Lumière Lyon 2, 29 septembre - 2 octobre 1994. Lyon, France: CRDP.

Mondada, L. \& Pekarek Doehler, S. (2001). Interactions acquisitionnelles en contexte: Perspectives théoriques et enjeux didactiques. Le français dans le monde, numéro spécial, 107-142.

Mondada, L. \& Pekarek Doehler, S. (2004). Second language acquisition as situated practice: task accomplishment in the French second language classroom. The Modern Language Journal 88(4), 501-518.

Mondada, L. \& Pekarek Doehler, S. (Eds.) (2006): La notion de compétence: études critiques. Bulletin suisse de linguistique appliquée VALS/ASLA, 84.

Moore, D. \& Simon, D. L. (2002). Déritualisation et identité d'apprenants. Acquisition et interaction en langue étrangère, 16, 1-19. Repéré à https://aile.revues.org/1374

Nunan, D. (2004). Task-based language teaching. Cambridge, UK: Cambridge University Press.

Pekarek Doehler, S. (2000). Approches interactionnistes de l'acquisition des langues étrangères: Concepts, recherches, perspectives. Acquisition et interaction en langue étrangère, 12, 3-26. Repéré à http://aile.revues.org/934?lang=en

Pekarek Doehler, S. (2002). Situer l'acquisition des langues secondes dans les activités sociales: l'apport d'une perspective interactionniste. Babylonia, 4, 24-29.

Pekarek Doehler, S. (2005). De la nature située des compétences en langue. Dans J. P. Bronckart, E. Bulea, \& M. Pouliot (Eds.), Repenser l'enseignement des langues: comment identifier et exploiter les compétences (pp. 41-68). Villeneuve d'Ascq: Presses universitaires du Septentrion.

Pekarek Doehler, S. (2006). Compétence et langage en action. Bulletin suisse de linguistique appliquée, 84, 9-45.

Pennings, H. J. M., Brekelmansa, M., Sadlerb, P., Claessens, L. C. A., van der Want, A. \& van Tartwijk, J. (2018). Interpersonal adaptation in teacher-student interaction.

Learning and Instruction, 55, 41-57.

Perdue, C. (1995). L'acquisition du français et de l'anglais par les adultes: Former des 
énoncés. Paris, France: CNRS Éditions.

Porquier, R. \& Py, B. (2004). Apprentissage d'une LE: contextes et discours. Paris, France: Didier.

Pochon-Berger, E. (2011). Analyse conversationnelle comme approche "sociale" de l'acquisition des langues secondes: une illustration empirique. TRANEL, 53, 122141.

Py, B. (1991). Bilinguisme, exolinguisme et acquisition: Rôle de L1 dans l'acquisition de L2. TRANEL, 17, 147-161.

Rogoff, B. (1990). Apprenticeship in learning. Oxford, UK: Oxford University Press.

Sabatier Bullock, C. (sous presse). Didactique du français et agir enseignants en contexte minoritaire britanno-colombien. Dans J. Thibeault \& C. Fleuret (Eds.), Didactique du français en contextes minoritaires: enjeux actuels et regards prospectifs. Ottawa, ON: Presses de 1'Université d'Ottawa.

Schegloff, E. (2006). Interaction: The infrastructure for social institutions, the natural ecological niche for language, and the arena in which culture is enacted. Dans N.J. Enfield \& S. Levinson (Eds), Roots of human sociality (pp. 70-96). Oxford, UK: Berg.

Skogmyr Marian, K., Petitjean, C. \& Pekarek Doehler S. (2017). Le développement de la compétence d'interaction en langue seconde : état des lieux et illustrations empiriques. Revue française de linguistique appliquée, 22(2), 127-145.

Swain, M. (2001). Intergrating language and content teaching through collaborative tasks. La Revue canadienne des langues vivantes, 58, 44-63.

Swain, M. \& Lapkin, S. (1998). Interaction and second language learning: Two adolescent French immersion students working together. The Modern Journal of Language, 83, 320-337.

Trévisiol, P. (2006). Influence translinguistique et alternance codique en français L3. Rôles des L1 et L2 dans la production orale d'apprenants japonais. Acquisition et interaction en langue étrangère, (24), 13-43.

Turnbull, B. (2016). Reframing foreign language learning as bilingual education: Epistemological changes towards the emergent bilingual. International Journal of Bilingual Education and Bilingualism 21(8), 1041-1048.

Véronique, D. (2007). L'apport des recherches sur l'acquisition des langues étrangères. Le Français dans le monde, 41, 96-106.

Vogel, K. (1995). L'interlangue, la langue de l'apprenant. Toulouse, France: Presses Universitaires du Mirail.

Walsh, S. (2006). Investigating classroom discourse. London, UK: Routledge.

Walsh, S. (2011). Exploring classroom discourse: Language in action. London, UK: Routledge.

Wolff, D. (2003). Integrating language and content in the language classroom: Are transfer of knowledge and of language ensured?. ASp. la revue du GERAS, 41-42, $35-46$.

Young, R. F. (2003). Learning to talk the talk and walk the walk: Interactional competence in academic spoken English. North Eastern Illinois University Working Papers in Linguistics, 2, 26-44. 


\section{Biographie de l'auteur}

Dr. Cécile Sabatier Bullock est professeur agrégée (Associate Professor) à la Faculté d'Éducation de Simon Fraser University. Ses travaux de recherche se situent dans les champs de l'Éducation plurilingue et interculturelle, de la sociolinguistique et de la didactique du plurilinguisme, et de la formation des enseignants dans des contextes de diversité linguistique et culturelle.sabatier@asfu.ca 